\title{
OXIMETRIA DE PULSO EM TRIAGEM DE CARDIOPATIAS CONGÊNITAS: CONHECIMENTO E ATUAÇÃO DO ENFERMEIRO*
}

\author{
Ana Lúcia de Medeiros ${ }^{1}$, Talita Bezerra Freitas², Juliana Sousa Soares de Araújo 3 , Sandra da Silva Mattos ${ }^{4}$
}

${ }^{1}$ Enfermeira. Doutoranda em Enfermagem. Docente de Enfermagem da Faculdade Internacional da Paraíba. João Pessoa, PB, Brasil.

${ }^{2}$ Discente de Enfermagem. Faculdade Internacional da Paraíba. João Pessoa, PB, Brasil.

${ }^{3}$ Médica. Mestre em Modelos de Decisão e Saúde. Docente da Faculdade de Ciências Médicas. João Pessoa, PB, Brasil.

${ }^{4}$ Médica. Doutora em Biotecnologia. Real Hospital Português de Beneficência em Pernambuco. João Pessoa, PB, Brasil.

RESUMO: A oximetria de pulso nas unidades neonatais apresenta-se como um processo de triagem de cardiopatias congênitas graves. O estudo tem como objetivo avaliar o nível de informação e a atuação dos enfermeiros na oximetria de pulso realizada nos recém-nascidos de uma maternidade pública do município de João Pessoa, Paraíba. Trata-se de estudo descritivo com abordagem quanti-qualitativa, realizado com 13 enfermeiras assistenciais através de entrevista semiestruturada no período de janeiro a março de 2015. Os resultados mostraram que a maioria das enfermeiras tem conhecimento sobre o teste do coraçãozinho, a justificativa para realizá-lo, os parâmetros de normalidades da saturação de oxigênio, assim como as condutas que devem ser tomadas diante de um resultado alterado. No entanto, 84,6\% referiram dificuldades na sua implantação em virtude de modificar a rotina da assistência de enfermagem. Acredita-se que o teste do coraçãozinho para triagem de cardiopatias congênitas traz bons resultados na captação precoce dessas malformações.

DESCRITORES: Enfermagem; Cardiopatia; Oximetria de pulso.

\section{PULSE OXIMETRY IN TRIAGING CONGENITAL CARDIOPATHIES: THE NURSE'S KNOWLEDGE AND ROLE}

ABSTRACT: Pulse oximetry in the neonatal units is presented as a process for triaging serious congenital cardiopathies. The study aims to assess the level of information, and the work, of the nurses in pulse oximetry undertaken on the newborns in a public maternity unit in the municipality of João Pessoa, Paraíba. It is a descriptive study with a quantiqualitative approach, undertaken with 13 staff nurses through the use of a semistructured interview in January - March 2015. The results showed that the majority of the nurses have knowledge regarding the 'little heart test' (teste do coraçãozinho), the rationale for undertaking it, and the normal parameters for oxygen saturation, as well as the conducts to be taken in the event of a result outside these parameters. However, $84.6 \%$ mentioned difficulties in implementing it due to having to alter the routine of the nursing care. It is believed that the little heart test for triaging congenital cardiopathies brings good results in the early identification of these malformations.

DESCRIPTORS: Nursing; Cardiopathies; Pulse oximetry.

\section{OXIMETRÍA DE PULSO EN SELECCIÓN DE CARDIOPATÍAS CONGÉNITAS: CONOCIMIENTO Y ACTUACIÓN DEL ENFERMERO}

RESUMEN: La oximetría de pulso en las unidades neonatales se presenta como un proceso de selección de cardiopatías congénitas severas. El estudio tiene como finalidad evaluar el nivel de información y la actuación de los enfermeros en la oximetría de pulso realizada en recién nacidos de una maternidad pública del municipio de João Pessoa, Paraíba. Es un estudio descriptivo con abordaje cuantitivo cualitativo, realizado con 13 enfermeras asistenciales a través de entrevista semiestructurada en el periodo de enero a marzo de 2015. Los resultados mostraron que la mayoría de las enfermeras tiene conocimiento sobre el test del corazoncito, la justificativa para realizarlo, los parámetros de normalidad de la saturación de oxígeno, así como las conductas delante de un resultado alterado. Sin embargo, $84,6 \%$ hablaron de dificultades en su implantación por el hecho de cambiarse la rutina de la asistencia de enfermería. Se cree que el test del corazoncito para selección de cardiopatías congénitas trae buenos resultados en la captación precoz de esas malas formaciones.

DESCRIPTORES: Enfermería; Cardiopatía; Oximetría de pulso.

*Artigo extraído da tese intitulada: "Análise de bancos de dados de um serviço de referência em Cardiologia Pediátrica e Fetal". Pós-graduação Programa da Universidade de Pernambuco, 2013.

Autor Correspondente:

Ana Lúcia de Medeiros

Faculdade Internacional da Paraíba

R. Juiz Arnaldo Ferreira Alves, 126 - 58052-315 - João Pessoa, PB, Brasil

E-mail: aninhapits@gmail.com
Recebido: $16 / 04 / 2015$

Finalizado: 13/08/2015 


\section{INTRODUÇÃO}

A evolução tecnológica aumentou exponencialmente o crescimento da indústria de computadores e aparelhos eletrônicos, tornando-se comum a utilização de diversos tipos de dispositivos em todas as partes do mundo inclusive no âmbito hospitalar. Presenciase, assim, uma cultura em que a tecnologia é imprescindível, e o homem por sua vez precisa aprender sobre essa nova realidade e adaptar-se a ela ${ }^{(1)}$.

Nesse sentido, a oximetria de pulso constitui um avanço tecnológico que permite a monitorização de forma contínua e não invasiva da saturação de oxigênio no sangue arterial, e que é utilizada como mais uma ferramenta na triagem neonatal e apresenta-se como possibilidade na redução da incidência de mortalidade e gravidade das complicações nos casos de cardiopatias congênitas ${ }^{(2)}$.

As cardiopatias congênitas são os principais problemas de morbimortalidade neonatal, atingindo aproximadamente oito a cada 1000 nascidos vivos ${ }^{(3)}$. Em torno de 30\% destes recém-nascidos recebem alta hospitalar sem o diagnóstico, e evoluem para choque, hipóxia ou óbito precoce, antes de receber tratamento adequado $^{(4)}$. Elas reúnem um conjunto variado de malformações cardíacas, com aspectos fisiológicos diferentes, e algumas delas, classificadas como malformações congênitas graves, necessitam de diagnóstico ainda nos primeiros dias de vida devido à sua rápida evolução, podendo ocasionar óbito precoce ${ }^{(5)}$.

Melhorar o diagnóstico destas cardiopatias poderá reduzir a taxa de mortalidade neonatal em nosso meio. $\mathrm{O}$ método ideal para o diagnóstico de cardiopatia congênita é o ecocardiograma com mapeamento de fluxo em cores no período fetal ou pós-natal ${ }^{(6)}$. Contudo, esse exame necessita de custos relevantes além de profissionais especializados, dificultando o diagnóstico precoce das cardiopatias congênitas mais graves ${ }^{(5)}$.

No grupo dessas cardiopatias, ocorre uma mistura de sangue entre as circulações sistêmica e pulmonar, o que acarreta uma redução da saturação periférica de O2. Neste sentindo, a aferição da oximetria de pulso de forma rotineira em recém-nascidos aparentemente saudáveis com idade gestacional $>34$ semanas, tem mostrado uma elevada sensibilidade e especificidade para detecção precoce dessas cardiopatias ${ }^{(7)}$.
No entanto, é necessário levar em consideração que a avaliação clínica do enfermeiro e do médico são também muito valiosas na identificação de anormalidades, inclusive cardiopatias, entre recém-nascidos.

Nesse sentido, um estudo multicêntrico realizado no Estado da Paraíba em 2012, mostra a grande relevância da avaliação clínica associada à oximetria de pulso e ao ecocardiograma de triagem. Foram realizadas 7026 oximetrias e 119 pacientes foram encaminhados para um ecocardiograma. Destes $10,1 \%$ apresentaram cardiopatia complexa, 14,3\% cardiopatia de shunt, $3,4 \%$ cardiopatia obstrutiva, $26,9 \%$ circulação transicional, 2,5\% alterações não estruturais e $42,9 \%$ tiveram eco normal. A indicação para o ecocardiograma foi clínica em $79 \%$ e baseada na oximetria, isoladamente, em $21 \%$. Apenas dois pacientes tiveram diagnóstico pré-natal de cardiopatia congênita ${ }^{(8)}$.

Baseada nessa realidade, a utilização da oximetria de pulso nas unidades neonatais apresenta-se como mais um processo de triagem de cardiopatias congênitas graves, de forma viável em relação a custos e a profissionais que possam realizá-la de forma eficaz. Conhecido como teste do coraçãozinho, este método tem obtido bons resultados na captação precoce dessas malformações.

Nesse contexto, o enfermeiro é o profissional ideal de escolha para a realização da triagem neonatal. Com informação e conhecimento frente ao manuseio correto das oximetrias de pulso, ele pode otimizar e ajudar na qualidade e eficácia deste processo.

Logo, o estudo tem como objetivo avaliar o nível de informação e a atuação dos enfermeiros na oximetria de pulso, realizado nos recém-nascidos de uma maternidade pública do município de João Pessoa, Paraíba.

\section{METODOLOGIA}

O estudo foi do tipo descritivo com abordagem quanti-qualitativa, realizado no período de janeiro a março 2015, em uma maternidade de referência no Estado, localizada no município de João Pessoa, Paraíba.

A população foi composta por enfermeiras que atuavam nos setores que prestam assistência aos recém-nascidos da maternidade em estudo. A amostra foi constituída por 13 enfermeiras que aceitaram participar voluntariamente da 
pesquisa, após serem devidamente informadas a respeito do objetivo e assinarem o termo de consentimento livre e esclarecido.

A construção dos dados foi realizada a partir de um roteiro de entrevista, semiestruturado, composto de duas partes. A primeira parte constou dos dados referentes às características do perfil das enfermeiras que realizam o teste do coraçãozinho, destacando as seguintes variáveis: faixa etária, o tempo de graduação e de serviço na instituição, a pós-graduação que o enfermeiro realizou e o setor de atuação.

A segunda parte refere-se ao nível de informação e a atuação das enfermeiras frente ao teste do coraçãozinho, atendendo assim o objetivo da pesquisa. Nessa parte, questionouse, de forma aberta, o que as enfermeiras entendiam sobre o teste do coraçãozinho e se elas conheciam a justificativa para realizá-lo. Em seguida, investigou-se as dificuldades na implantação do teste no serviço, os parâmetros ideais da oximetria de pulso e a conduta que deveria ser tomada diante de uma alteração do mesmo. Essas questões mostravam alternativas de escolha para que a enfermeira optasse pela melhor resposta.

A compilação dos dados quantitativos foi realizada no Microsoft Excel e apresentada em gráficos e tabela. As variáveis quantitativas foram submetidas a medidas descritivas $e$ distribuições de frequência. Para a análise dos dados qualitativos, empregou-se a técnica do Discurso do Sujeito Coletivo (DSC), que consiste num conjunto de procedimentos que destaca as expressões chave das falas das participantes do estudo, o que viabiliza o pensamento em forma de síntese e possibilita a interpretação para fundamentação dos resultados ${ }^{(9)}$.

Este estudo adotou, em todas as suas fases, as diretrizes e normas regulamentadoras de pesquisas envolvendo seres humanos que constam na Resolução 466/12 do Conselho Nacional de Saúde ${ }^{(10)}$ e foi aprovado pelo Comitê de Ética em Pesquisa do Hospital Universitário Oswaldo Cruz - HUOC/PROCAPE da Universidade de Pernambuco, sob o parecer de n 391/391 de 06/09/2013.

\section{RESULTADOS}

Os dados obtidos são apresentados em três momentos: o primeiro mostra a caracterização das enfermeiras que realizam a oximetria de pulso (Tabela 1); o segundo, no qual são apresentados
Tabela 1 - Perfil profissional das enfermeiras que realizam a oximetria de pulso. João Pessoa- PB, Brasil, 2015

\begin{tabular}{|c|c|c|}
\hline Perfil Profissional & $\mathrm{N}=13$ & $\%$ \\
\hline \multicolumn{3}{|l|}{ Faixa Etária } \\
\hline 21-30 anos & 1 & 8 \\
\hline 31- 40 anos & 9 & 69 \\
\hline 41-50 anos & 1 & 8 \\
\hline 51-60 anos & 2 & 15 \\
\hline \multicolumn{3}{|l|}{ Tempo de Graduação } \\
\hline $1-5$ anos & 3 & 24 \\
\hline 6-10 anos & 5 & 38 \\
\hline 11-15 anos & 2 & 15 \\
\hline 16-20 anos & 1 & 8 \\
\hline 21-25 anos & 2 & 15 \\
\hline \multicolumn{3}{|l|}{ Tempo de Serviço na Instituição } \\
\hline $0-5$ anos & 10 & 77 \\
\hline $6-10$ anos & 2 & 15 \\
\hline 11-15 anos & 1 & 8 \\
\hline \multicolumn{3}{|l|}{ Pós-Graduação (lato sensu) } \\
\hline Sim & 10 & 77 \\
\hline Não & 3 & 23 \\
\hline \multicolumn{3}{|l|}{ Área da Pós-Graduação* } \\
\hline Saúde Pública & 2 & 20 \\
\hline Urgência e Emergência & 1 & 10 \\
\hline $\begin{array}{l}\text { Saúde Pública e Enfermagem do } \\
\text { Trabalho }\end{array}$ & 3 & 30 \\
\hline Outras & 4 & 40 \\
\hline \multicolumn{3}{|l|}{ Setor de Atuação } \\
\hline Alojamento conjunto & 10 & 77 \\
\hline Enfermaria canguru** & 1 & 8 \\
\hline Triagem & 1 & 8 \\
\hline $\mathrm{CCIH}^{* * *}$ & 1 & 8 \\
\hline
\end{tabular}

*A área de pós-graduação somou 10 respostas devido a 3 enfermeiras não possuírem pós-graduação.

**A enfermaria canguru é o setor onde funciona o Método Mãe-Canguru que é um tipo de assistência neonatal baseada no contato pele a pele, precoce e progressivo, entre pai, mãe e bebê até se atingir a posição canguru.

***CCIH: Comissão de Controle de Infecção Hospitalar.

os dados qualitativos da pesquisa, referente ao conhecimento das enfermeiras sobre a oximetria de pulso; o terceiro, os dados quantitativos, referentes às dificuldades de implantação do teste, os parâmetros de normalidades e as condutas a serem tomadas pelas enfermeiras diante de um resultado alterado.

Pode-se observar na Tabela 1 que a faixa etária predominante das enfermeiras foi entre 31 a 40 anos de idade, correspondente a $69 \%$. O tempo de formação acadêmica variou de 01 a 25 
anos, com maior prevalência entre 6 a 10 anos, correspondendo a $38 \%$, e o tempo de serviço na instituição variou entre 0 e 15 anos, com $77 \%$ das entrevistadas no período de 0 a 5 anos de atuação na instituição. Em relação à pós-graduação lato sensu, $77 \%$ das enfermeiras entrevistadas afirmaram ter algum tipo de especialização, sendo $30 \%$ em saúde pública e enfermagem do trabalho e $40 \%$ em outras áreas não especificadas. O setor de atuação de maior prevalência entre as entrevistadas foi no alojamento conjunto, correspondente a $77 \%$, com o binômio mãe e filho recebendo os primeiros cuidados, incluindo o teste do coraçãozinho, realizado pela enfermeira.

Quanto ao conhecimento das enfermeiras sobre o teste do coraçãozinho, são apresentados os questionamentos a seguir:

\section{O que você entende por teste do coraçãozinho?}

A partir das respostas a este questionamento, extraíram-se duas ideias centrais. A primeira foi sobre a triagem neonatal de cardiopatias congênitas graves e a segunda sobre o teste realizado através da oximetria de pulso.

É um teste de triagem realizado nos recémnascidos, nas primeiras 24 horas de vida através da oximetria de pulso, na qual se avalia o nível de saturação de oxigênio e a frequência cardíaca com a finalidade de detectar precocemente cardiopatias congênitas graves, evitando-se dessa forma, a morte súbita e prematura em recém-nascidos (DSC 1).

\section{Qual a justificativa para realizar o teste do coraçãozinho?}

Surgiram dos discursos das enfermeiras as seguintes ideias centrais: rastrear cardiopatias congênitas graves, iniciar tratamento precocemente e diminuir a mortalidade infantil.

A análise das falas possibilitou a construção do segundo Discurso do Sujeito Coletivo, expresso a seguir:

A realização do teste do coraçãozinho através da oximetria de pulso se faz necessária no rastreamento de cardiopatias congênitas graves a fim de iniciar o tratamento específico o mais rápido possível, evitando complicações mais sérias e diminuindo a mortalidade infantil (DSC 2).

Os dados mostraram ainda que $77 \%$ das enfermeiras referiram não ter conhecimento ou não ter ouvido falar sobre o teste do coraçãozinho, antes de sua implantação na unidade hospitalar, apenas $23 \%$ referiram ter ouvido falar sobre o teste através da internet.
Outro questionamento realizado com as enfermeiras foi se elas sentiram dificuldades na implantação do teste do coraçãozinho no serviço. As entrevistadas, em sua maioria $(85 \%)$ referiram dificuldades na inserção do teste na rotina da assistência de enfermagem. As dificuldades relatadas encontram-se ilustradas na Figura 1.

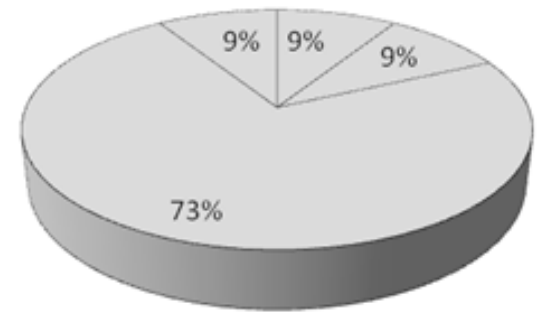

$73 \%$ - Acúmulo de tarefa e falta de treinamento

9\% - Acúmulo de tarefa

$9 \%$ - Falta de treinamento

$9 \%$ - Outros

Figura 1 - Dificuldades na implantação do teste do coraçãozinho. João Pessoa-PB, Brasil, 2015

A Figura 1 mostra que o acúmulo de tarefas associadoà falta de treinamento foram as principais dificuldades enfrentadas pelas enfermeiras na implantação do teste do coraçãozinho na rotina da assistência de enfermagem, correspondendo a $73 \%$ das dificuldades citadas.

Continuando a análise quantitativa, foi questionado se as enfermeiras conheciam os parâmetros de normalidades da oximetria de pulso realizada nos recém-nascidos recomendados pelo Ministério da Saúde, que é a saturação periférica maior ou igual a $95 \%$ em ambas as medidas (membro superior direito e membro inferior) e diferença menor que $3 \%$ entre as medidas do membro superior direito e membro inferior.

A maioria (85\%) das entrevistadas sabia identificar os parâmetros de normalidades de acordo com a norma proposta pelo Ministério da Saúde. A maioria (73\%) das enfermeiras reconhece que a saturação periférica maior ou igual a $95 \%$ em ambas as medidas (membro superior direito e membro inferior) e diferença menor que 3\% entre as medidas do membro superior direito e membro inferior é considerado o padrão ideal pelo Ministério da Saúde. No último questionamento foi perguntado se conheciam a conduta a ser tomada ao identificar alteração no resultado do teste do coraçãozinho.

Das 13 enfermeiras que fizeram parte da amostra, 11 responderam que conheciam a conduta que o profissional de saúde deveria tomar. 


\section{DISCUSSÃO}

Analisando as falas, sintetizadas por meio do Discurso do Sujeito Coletivo 1, foi possível perceber que as enfermeiras sabiam informar o que era o teste do coraçãozinho. Assim como, através do Discurso do Sujeito Coletivo 2, elas relataram que o objetivo do teste do coraçãozinho era ajudar a detectar cardiopatias congênitas precoces e consequentemente contribuir para a diminuição da morbimortalidade infantil.

O componente neonatal da mortalidade infantil está estreitamente vinculado aos cuidados no período da gestação, do nascimento e do recém-nascido. Implica, portanto, a atenção adequada no momento do nascimento e os cuidados destinados aos recém-nascidos, com práticas simples, baratas e baseadas em evidências científicas que aumentam os índices de sobrevivência dos recém-nascidos ${ }^{(7)}$. Destas práticas, destaca-se o teste do coraçãozinho, que objetiva triar cardiopatias congênitas graves.

São consideradas cardiopatias congênitas graves aquelas nas quais a apresentação clínica decorre do fechamento ou restrição do canal arterial (cardiopatias canal-dependentes), tais como: cardiopatias com fluxo pulmonar dependente do canal arterial: atresia pulmonar e similares; cardiopatias com fluxo sistêmico dependente do canal arterial: síndrome de hipoplasia do coração esquerdo, coarctação da aorta críticas e similares; cardiopatias com circulação em paralelo: transposição das grandes artérias $^{(6)}$.

No grupo das cardiopatias congênitas graves, ocorre uma mistura de sangue entre as circulações sistêmica e pulmonar, o que acarreta uma redução da saturação periférica de oxigênio. Desse modo, a justificativa para a realização da oximetria de pulso como método de rastreio de cardiopatias congênitas graves se dá pelo fato de haver hipóxia mesmo antes da cianose ser evidenciada ${ }^{(11)}$. Neste sentido, a aferição da oximetria de pulso de forma a triar cardiopatias congênitas apresenta-se como um teste satisfatório e indicador de anormalidades cardíacas.

Os dados da pesquisa mostraram também que a maioria das enfermeiras sabe interpretar o resultado do teste do coraçãozinho, apontando os parâmetros considerados ideais recomendados pelo Ministério da Saúde, assim como tem conhecimento das condutas a serem tomadas diante de uma alteração na oximetria de pulso.
O Ministério da Saúde considera um resultado normal quando a saturação periférica for maior ou igual a 95\% em ambas as medidas (membro superior direito e membro inferior) e diferença menor que $3 \%$ entre as medidas do membro superior direito e membro inferior. Caso qualquer medida da $\mathrm{SpO} 2$ seja menor que $95 \%$ ou houver uma diferença igual ou maior que $3 \%$ entre as medidas do membro superior direito e membro inferior, uma nova aferição deverá ser realizada após 1 hora. Caso o resultado se confirme, um ecocardiograma deverá ser realizado dentro das 24 horas seguintes ${ }^{(12)}$.

O método ideal para fechar o diagnóstico de cardiopatia congênita é o ecocardiograma com mapeamento de fluxo em cores, que se torna inviável na rotina de todos os nascidos vivos, devido ao seu custo e manuseio especializado. Dessa forma, a triagem neonatal de cardiopatias congênitas através da oximetria de pulso permite selecionar os recém-nascidos aparentemente saudáveis, que teriam alta hospitalar dentro de 36 a 48 horas $^{(6)}$.

No entanto, a avaliação clínica contínua dos profissionais de saúde é fundamental, destacandose a Sistematização da Assistência de Enfermagem para os enfermeiros, na qual são implementadas ações de rotina que identificam todo o conjunto e as particularidades de cada paciente.

A oximetria de pulso constitui uma inovação tecnológica, que utiliza a absorção da luz vermelha e infravermelha pela hemoglobina oxigenada. A interpretação da saturação de $\mathrm{O} 2$ deve levar em consideração vários fatores, como: má posição do sensor, artefatos por movimento, luz ambiente, temperatura, entre outros ${ }^{(3)}$. É um método não invasivo, indolor e rápido de realizar. A realização deste teste não descarta a necessidade de realização de exame físico completo e minucioso, além de ausculta cardíaca antes da alta hospitalar.

Nesse cenário, o enfermeiro é o profissional indicado na realização do teste do coraçãozinho, pois é responsável pelos cuidados primários realizados ao binômio mãe e filho no puerpério. Sendo assim, sua atuação no processo de triagem de cardiopatias congênitas potencializa a qualidade da assistência aos recém-nascidos.

Observa-se que a Lei do Exercício Profissional, Lei $\mathrm{N}^{\circ} 7.498 / 86^{(13)}$ e o Decreto $\mathrm{N}^{\circ}$ 94.406/87 $7^{(14)}$, em seu artigo $8^{\circ}$, asseguram ao profissional enfermeiro a competência para executar procedimentos de Enfermagem complexos em recém-nascidos, com cuidados de enfermagem de maior complexidade 
técnica que exijam conhecimentos científicos adequados e capacidade de tomar decisões, situação que caracteriza a oximetria de pulso na Triagem Neonatal, conforme a Portaria No 20/2014 do Ministério da Saúde ${ }^{(15)}$.

No cenário onde a pesquisa foi realizada, maternidade de referência em ginecologia e obstetrícia do estado, teve o teste do coraçãozinho implantado nas unidades neonatais a partir de 2014, através da Portaria $\mathrm{n}^{\circ} 20$, de 10 de junho de 2014, a qual se torna pública a decisão de incorporar a oximetria de pulso - teste do coraçãozinho, a ser realizado de forma universal, fazendo parte da triagem neonatal do Sistema Único de Saúde (SUS).

Nesse contexto, a oximetria de pulso constitui medida importante na assistência de enfermagem ao recém-nascido na triagem neonatal de cardiopatias congênitas, podendo ser realizada de forma contínua sem custos excessivos. É uma atribuição relevante do enfermeiro, que deve garantir o registro adequado das variações da oximetria, como também saber correlacionar as suas anormalidades dentro dos parâmetros estabelecidos pelo Ministério da Saúde.

Logo, o conhecimento do enfermeiro na realização do teste do coraçãozinho é de grande importância no que diz respeito às etapas envolvidas na triagem de cardiopatias congênitas neonatais. O seu nível de conhecimento sobre o assunto e atenção rigorosa às alterações do teste garantem que as tomadas de decisões aconteçam de forma correta e contribuam de forma eficaz para o rastreio de cardiopatias congênitas graves.

Frente ao exposto, não há obstáculo da realização da oximetria de pulso na triagem neonatal pelo enfermeiro, uma vez que ele tem formação técnica especializada, compatível com a realização do procedimento devendo ser observadas as normas, rotinas e protocolos de atendimento que regem o exercício da profissão de Enfermagem.

\section{CONSIDERAÇÕES FINAIS}

Os resultados revelaram que as enfermeiras sabem o conceito e o objetivo do teste do coraçãozinho, assim como sabem descrever como este é aplicado na prática da enfermagem por meio da verificação de saturação de oxigênio, com a finalidade de triar possíveis cardiopatias congênitas e assim reduzir o índice de mortalidade infantil.
Oestudo mostrou ainda que um grande número de enfermeiras sentiu dificuldades no momento de implantação do teste no serviço, uma vez que não tinha conhecimento sobre o funcionamento do mesmo e não houve treinamento para sua implantação, somado às inúmeras tarefas que já fazem parte do cotidiano do enfermeiro no setor do alojamento conjunto.

Outro dado importante que a pesquisa evidenciou foi que um número bastante expressivo de enfermeiras sabe identificar os parâmetros de normalidade da oximetria de pulso em recém-nascido e as condutas a serem tomadas diante de um resultado alterado.

Nesse contexto, percebe-se a importância da atuação do enfermeiro na realização do teste do coraçãozinho nas unidades neonatais. No entanto, os estudos envolvendo essa temática ainda são muito incipientes, necessitando de mais pesquisa para demonstrar os benefícios desse papel que o enfermeiro vem assumindo com a implantação do programa de triagem neonatal, especificamente com o teste do coraçãozinho.

Logo, o estudo assinala que o conhecimento e o nível de informação desses profissionais sobre cardiopatias congênitas e sobre o teste do coraçãozinho podem refletir diretamente nas tomadas de decisões referentes aos parâmetros do teste, possibilitando o manuseio correto dos recém-nascidos no processo de triagem de anormalidades cardíacas.

\section{REFERÊNCIAS}

1 Júnior DFM, Guastelli LR, França SR. Enfermagem e informática. In: Knobel, E. Terapia Intensiva: Enfermagem. São Paulo: Atheneu; 2010. p. 49-55.

2 Borges JPA. Monitorização da oximetria de pulso em recém-nascidos: atuação do enfermeiro nas unidades neonatais. Rev. enferm. atenção saúde. [Internet] 2013;2(3)[acesso em 04 mar 2015]. Disponível: http:// www.uftm.edu.br/revistaeletronica/index.php/enfer/ article/view/595/440

3 Morais S, Mimoso G. Oximetria de pulso no diagnóstico de cardiopatia congênita: sugestões para a implementação de uma estratégia de rastreio. Acta pediatr. port.[Internet] 2013;44(6) [acesso em 02 mar 2015]. Disponível: http://actapediatrica.spp.pt/article/ view/2717/2758

4Mellander M, Sunnegardh J. Failureto diagnose critical heart malformations in new borns before discharge - an increasing problem? Acta paediatr. [Internet] 2006;95(4) [acesso em 21 mar 2015]. Disponível: http:// www.ncbi.nlm.nih.gov/pubmed/16720486 
5 Moser LRDN, Diogenes TCP, Souza VOP, Oliveira ARF, Mourato FA, Mattos SS et.al. Novo modelo de teletriagem das cardiopatias congênitas. J bras. tele. [Internet] 2014;3(1) [acesso em 13 abr 2015]. Disponível: http://www.e-publicacoes.uerj.br/index. php/jbtelessaude/article/view/10236/8024

6 Sociedade Brasileira de Pediatria (SBP). Diagnóstico precoce de cardiopatia congênita crítica: oximetria de pulso como ferramenta de triagem neonatal. Departamentos de Cardiologia e Neonatologia. [Internet] 2011 [acesso em 30 mar 2015]. Disponível: http://www.sbp.com.br/pdfs/diagnostico-precoceoximetria.pdf

7 Kemper AR, Mahle WT, Martin GR, Cooley C, Kumar $\mathrm{P}$, Morrow R et al. Strategies for

implementing screening for critical congenital heart disease. Pediatrics. [Internet]2011;128 (5)[acesso em 30 mar 2015]. Disponível: http://pediatrics. aappublications.org/content/128/5/e1259.full

8 Régis CT, Soares JS, Gomes RGS, Silva CS, Lofiego P, Melo F. Realização de eco de triagem pelo neonatologista: experiência inicial. In: 670 Congresso Brasileiro de Cardiologia, 2012, Recife-PE. Arquivos Brasileiros de Cardiologia, 2012.

9 Lefèvre F, Lefèvre AM. Depoimentos e discursos: uma proposta de análise em pesquisa social. Brasília: Liber Livros Editora; 2005.

10 Ministério da Saúde (BR). Conselho Nacional de Saúde. Diretrizes e normas regulamentadoras de pesquisas envolvendo seres humanos. Resolução n. 466, de 12 de dezembro de 2012. Brasília; 2012 [acesso em 10 abr 2015]. Disponível: http://www.conselho. saude.gov.br/web_comissoes/conep/index.html

11 Mattos SS. Cardiologia para o pediatra: manual prático para o reconhecimento e manuseio das cardiopatias na infância. Recife: Série CADUCEUS; v1, 2004.

12 Ministério da Saúde (BR), Secretaria de Ciência, Tecnologia e Insumos Estratégicos. Comissão Nacional de Incorporação de Tecnologias no SUS (CONITEC). Relatório $\mathrm{n}^{\circ} 115$, de 10 de junho de 2014, que dispõe sobre a oximetria de pulso - teste do coraçãozinho, a ser realizado de forma universal, fazendo parte da triagem neonatal. Brasília; 2014 [acesso em 05 abr 2015]. Disponível: http://conitec.gov.br/images/ Incorporados/TesteCoracaozinho-FINAL.pdf

13 Brasil. Lei n. 7.498, de 25 de junho de 1986. Dispõe sobre a Regulamentação do Exercício da Enfermagem e dá outras providências. Diário Oficial da República Federativa do Brasil, Brasília, 26 jun. 1986. Seção 1:1. Brasil. Constituição da República Federativa do Brasil. Brasília: Senado;1988.

14 Ministério da Saúde (BR). Decreto n94406, de 08 de junho de 1987, que regulamenta a Lei $n^{\circ} 7498$ do exercício profissional da Enfermagem e dá outras providências. Brasília; 1987 [acesso em 15 abr 2015]. Disponível: http://www.planalto.gov.br/ccivil_03/ decreto/1980-1989/D94406.htm

15 Ministério da Saúde (BR) - Secretaria de Ciência, Tecnologia e Insumos Estratégicos. Portaria $n^{\circ} 20$, de 10 de junho de 2014, que torna pública a decisão de incorporar a oximetria de pulso - teste do coraçãozinho, a ser realizado de forma universal, fazendo parte da triagem Neonatal no Sistema Único de Saúde - SUS. Brasília; 2014 [acesso em 05 abr 2015]. Disponível: http://bvsms.saude.gov.br/bvs/saudelegis/sctie/2014/ prt0020_10_06_2014.html 